\title{
A Building-Block Favoring Method for the Topology Optimization of Internal Antenna Design
}

\author{
Yen-Sheng Chen \\ Department of Electronic Engineering, National Taipei University of Technology, 1, Sec. 3, Zhongxiao E. Road, Taipei 10608, Taiwan \\ Correspondence should be addressed to Yen-Sheng Chen; yschen@ntut.edu.tw
}

Received 15 June 2015; Accepted 5 July 2015

Academic Editor: Jaume Anguera

Copyright ( 2015 Yen-Sheng Chen. This is an open access article distributed under the Creative Commons Attribution License, which permits unrestricted use, distribution, and reproduction in any medium, provided the original work is properly cited.

\begin{abstract}
This paper proposes a new design technique for internal antenna development. The proposed method is based on the framework of topology optimization incorporated with three effective mechanisms favoring the building blocks of associated optimization problems. Conventionally, the topology optimization of antenna structures discretizes a design space into uniform and rectangular pixels. However, the defining length of the resultant building blocks is so large that the problem difficulty arises; furthermore, the order of the building blocks becomes extremely high, so genetic algorithms (GAs) and binary particle swarm optimization (BPSO) are not more efficient than the random search algorithm. In order to form tight linkage groups of building blocks, this paper proposes a novel approach to handle the design details. In particular, a nonuniform discretization is adopted to discretize the design space, and the initialization of GAs is assigned as orthogonal arrays (OAs) instead of a randomized population; moreover, the control map of GAs is constructed by ensuring the schema growth based on the generalized schema theorem. By using the proposed method, two internal antennas are thus successfully developed. The simulated and measured results show that the proposed technique significantly outperforms the conventional topology optimization.
\end{abstract}

\section{Introduction}

Following the rapid development of wireless and portable communication products, it is of the utmost importance that the design cycle of antennas is reduced. Conventionally, antenna design relies on optimizing the geometric shape of an initial layout. That is, the design parameters of an antenna structure are first identified, and their values are fine-tuned via trial-and-error approaches, genetic algorithms (GAs) $[1$, $2]$, particle swarm optimization (PSO) [3, 4], space mapping [5], or artificial neural networks (ANN) [6]. However, such approaches may fail to produce a satisfactory design if the detailed initial guess is incorrect.

Instead of tuning the geometric parameters of a given initial layout, topology optimization, also known as pixelated design technique, conceptual design, and optimal material distribution, does not require a detailed predefined shape, yet it automatically generates a suitable antenna topology fulfilling design needs. It discretizes the design space into several rectangular pixels, varying the distribution of conductor until design goals are achieved. Topology optimization usually provides an unexpected antenna shape, thereby preventing designer's improper biases and developing innovative layouts. More importantly, topology optimization is particularly suitable for the internal antenna design of wireless and portable communication products, for the antenna design space in these products that are usually confined in a given space; hence, topology optimization shows great competence in practical design situations, leading to a shorter design cycle and a lower development cost.

Topology optimization was developed in the sphere of mechanical engineering from 1988 [7]. It was primarily applied to the electromagnetic (EM) engineering field in 1996 [8], and the first design instance was magnetic bearings with minimum power dissipation. After that, topology optimization was implemented for antenna design problems [9]; it especially has gained much attention in the design of patch antennas [10-22] and monopole antennas [22-28]. However, only few studies have been done on other types of antennas such as chip antennas [29], planar-inverted-F antennas [30], handset antennas [31], and mobile antennas [32] using topology optimization. Furthermore, there has 


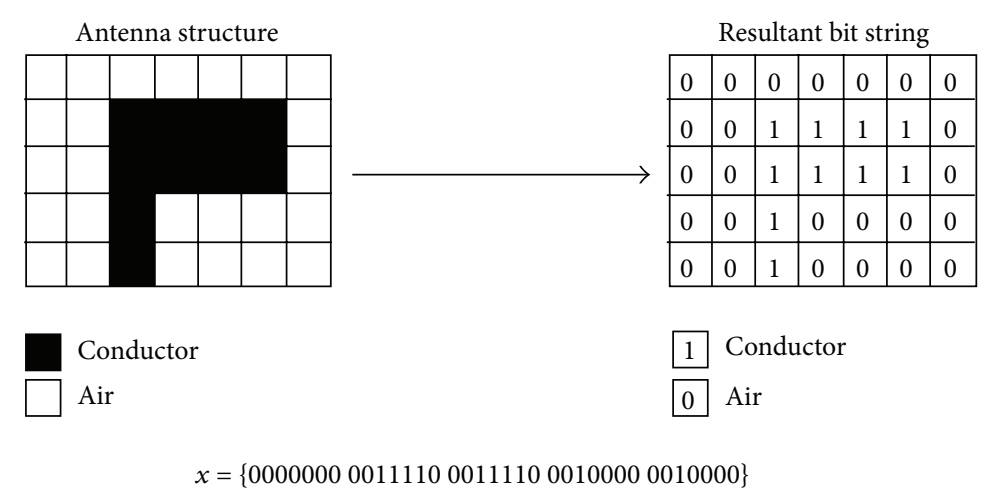

FIGURE 1: The relationship between the physical antenna structure and the resultant bit string.

been far less research on the characterization of problem difficulty for topology optimization. Most of the literature straightforwardly applied binary optimization algorithms because the design parameters of topology optimization problems are binary variables, but we lack a fundamental research showing that these binary optimization algorithms are indeed more effective than the random search algorithm. In fact, there is very limited study on analysis of the problem nature of topology optimization. Not until we fully investigate the problem difficulty can the optimization process take appropriate steps.

The purpose of this paper is to examine the problem nature of topology optimization and to propose a new design methodology based on what this problem really needs. More specifically, three classic antenna design problems are implemented to investigate the problem difficulty of topology optimization. The numerical results will show that, without understanding the structure of building blocks for these problems, binary optimization algorithms including GAs and binary PSO (BPSO) are not more efficient than the random search algorithm. Therefore, a new methodology is designed to cope with the structure of building blocks. This new building-block favoring method aims to lower the order of the building blocks and shorten the associated defining length; meanwhile, the initialization focuses on generating fairly distributed and diverse building blocks and parameterization concerns about the growth of schemata based on the generalized schema theorem. The mechanisms and the numerical results will be presented in detail. Furthermore, in order to validate the proposed method, two internal antenna design problems are addressed. The antenna performances supplemented with simulated and measured results will be exhibited.

\section{Characterization of Problem Difficulty}

Before elaborating the procedure of the building-block favoring method, this section presents the need of a new technique for topology optimization. Topology optimization discretizes a design space into several pixels; each pixel could fill with either conductor or air, so the design problem can be formulated as a binary programming problem:

$$
\text { Find: } \begin{array}{rl}
\mathbf{x}^{*}=\arg \min _{\mathbf{x}} & f(\mathbf{x}), \\
\text { Subject to: } & \left\{\begin{array}{l}
\mathbf{x} \in\{0,1\}, \\
\text { Problem constraints, }
\end{array}\right.
\end{array}
$$

where $\mathbf{x}$ is the $N$-vector of binary decision variables, representing the encoded states mapped from a specific antenna topology. The mapping procedure is shown in Figure 1; the pixels of the antenna structure filling with conductors are encoded as 1 , and the pixels filling with air are encoded as 0 . $f$ is the objective function, which is the function of the combination of decision variables. Topology optimization problem (1) belongs to the class of NP-hard problems, so only the enumeration method provides the global best solution; however, such time complexity is totally unacceptable, so nature-inspired heuristic algorithms, such as GAs [9-20, 23$27,29,30,32]$ and BPSO [21, 28, 31], are widely used to find a suboptimal solution. Nevertheless, it seems extremely premature to straightforwardly apply the binary optimization algorithms without specifying the problem nature.

In order to characterize the problem difficulty, three classic topology optimization problems for antenna design are investigated as follows. Each problem is addressed by three optimizers, including GAs, BPSO, and random search algorithms. In addition, the EM simulation is conducted by Ansoft HFSS.

2.1. Dual-Band Patch Antennas. The first instance to be investigated is a dual-band patch antenna for wireless communications [17]. The design environment is shown in Figure 2. Specifically, the patch antenna is fed by a 50 -ohm coaxial cable, and the upper patch, having an area of $36 \times 48 \mathrm{~mm}^{2}$, is discretized into 46 pixels. The substrate is a $15-\mathrm{mm}$-height FR4 (dielectric constant $\varepsilon_{r}=4.4$ and loss tangent $\tan \delta=$ $0.02)$. The design goal is to generate a dual-band operation at $1.9 \mathrm{GHz}$ and $2.4 \mathrm{GHz}$, so the objective function to be minimized is the maximum $\left|S_{11}\right|$ of $1.9 \mathrm{GHz}$ and $2.4 \mathrm{GHz}$. 


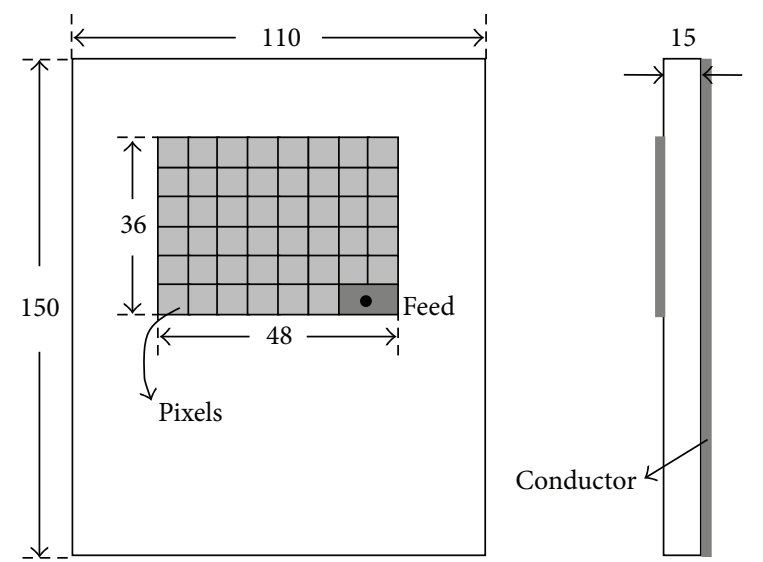

FIGURE 2: Geometry of the patch antenna and the discretization of the design space (unit: $\mathrm{mm}$ ).

The three optimizers mentioned above were applied to this problem. Unless otherwise noted, the following GA parameterization is applicable to all the three instances presented in this section: iterations $N_{\text {ite }}=30$, population size $N_{\text {pop }}=$ 120 , tournament selection with selection pressure $s=2$, replacement $r=0.1$, uniform crossover with a probability $p_{c}=0.5$, and mutation with a probability $p_{m}=0.01$. On the other hand, the BPSO parameterization used in the three examples is iterations $N_{\text {ite }}=30$, population size $N_{\text {pop }}=120$, fixed inertial weight $w=1$, fixed coefficients for global best attraction and personal best attraction $c_{1}=c_{2}=2$, and the limit of velocity $v_{\max }=6$. Lastly, the random search algorithm performs 3,600 looks, where the optimum solution is determined. Note that the three optimizers take the same number of functional evaluations. In addition, the termination criterion used in each case is time constraint. Once each optimizer reaches the maximum number of functional evaluations, namely, 3,600 looks, the optimization process will be terminated. More functional evaluations have been conducted after 3,600 looks, and a better performance is indeed observed; however, it is found that the improvement is very marginal and insignificant. Therefore, the total number of functional calls is set to 3,600 , and the best design among the 3,600 tests is selected and compared.

The performances of the optimized antenna structures are shown and compared in Figure 3. The GA found the optimum structure in the 27th generation, the BPSO obtained the optimum result in the 25th iteration, and the random search algorithm attained the optimum structure at the 3,504th look. Clearly, although the three optimizers obtained three different configurations, they provide satisfactory dual-band performances. However, no strong evidences show that the random search algorithm has inferior performances, and the efficiency of the binary optimization algorithms does not dominate that of the random search algorithm.

2.2. Dual-Band Monopole Antennas. Next, the problem difficulty of a dual-band monopole antenna [28] is investigated. The monopole antenna, shown in Figure 4, is designed for handheld devices. The design space is of area $36 \times 48 \mathrm{~mm}^{2}$,

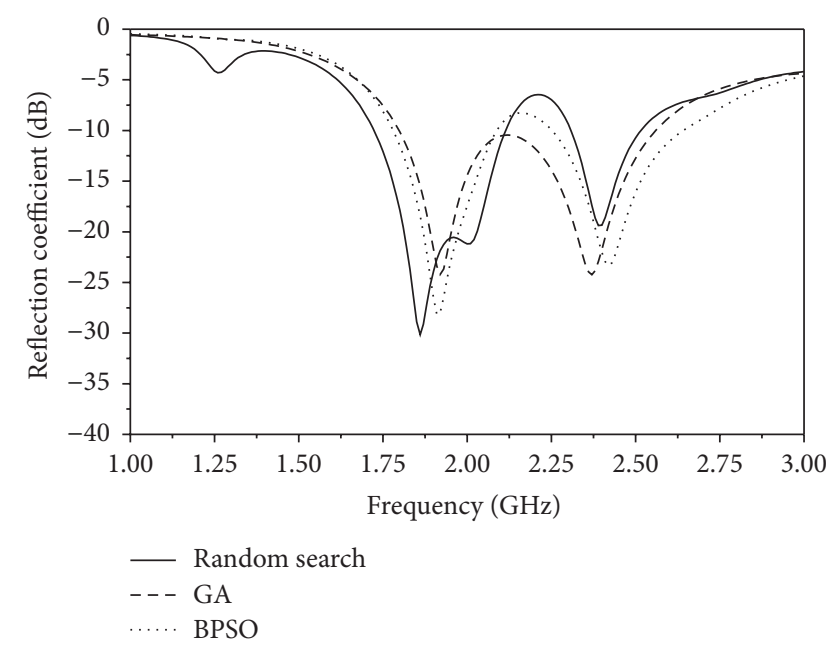

FIGURE 3: Simulated reflection coefficients of the optimized patch antennas corresponding to the GA, the BPSO, and the random search algorithm.

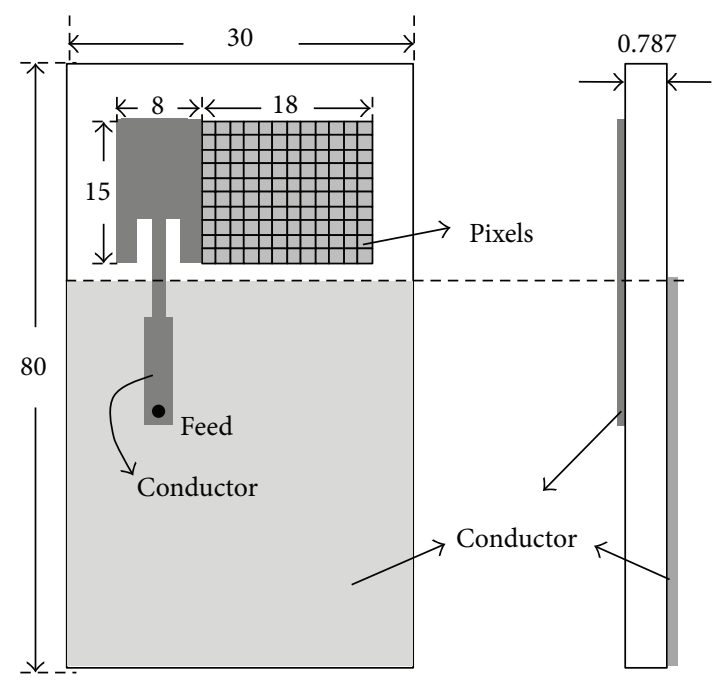

FIGURE 4: Geometry of the dual-band monopole antenna and the discretization of the design space (unit: $\mathrm{mm}$ ).

discretized into 120 rectangular pixels printed on a Rogers $\mathrm{RT} /$ Duroid 5880 dielectric substrate $\left(\varepsilon_{r}=2.2\right.$ and $\tan \delta=$ 0.0009 ) of thickness $0.787 \mathrm{~mm}$. In this case, the design objective is to have a dual-band performance at $1.8 \mathrm{GHz}$ and 2.4 GHz. Using the parameterization described above, the optimum structures can be evaluated after 3,600 functional evaluations.

The impedance matching of the optimum structures is exhibited in Figure 5. It can be seen that the performances between the three optimization algorithms are comparable. Specifically, the GA obtained the optimum structure in the 26th iteration, the BPSO found the optimum solution in the 28th iteration, and the random search algorithm determined the optimum structure at the 2,421st look. Once again, the efficiency of GAs and BPSO does not outperform that of 


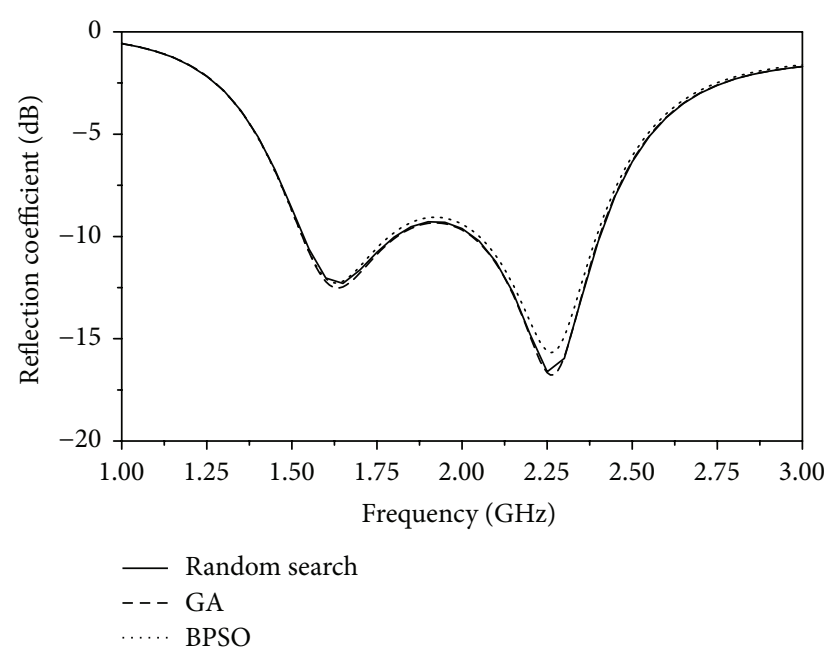

Figure 5: Simulated reflection coefficients of the optimized dualband monopole antennas corresponding to the GA, the BPSO, and the random search algorithm.

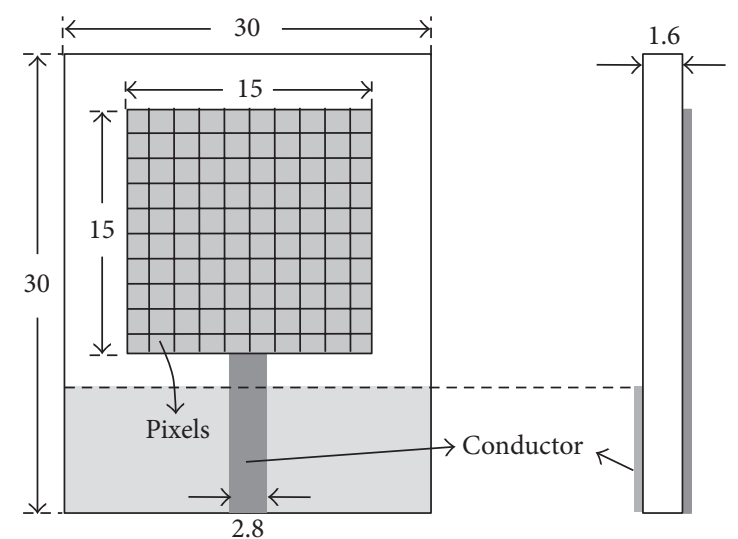

FIGURE 6: Geometry of the UWB monopole antenna and the discretization of the design space (unit: $\mathrm{mm}$ ).

the random search algorithm. On the other hand, increasing the population size of GAs and BPSO has similar results.

2.3. Ultra-Wideband Monopole Antennas. Lastly, let us consider the design problem of ultra-wideband (UWB) antennas [23-27]. A planar monopole structure shown in Figure 6 is chosen, and its square monopole patch is defined as the design space. The monopole is printed on a FR4 substrate of thickness $1.6 \mathrm{~mm}$, and the width of the feed line is $2.8 \mathrm{~mm}$. The design space is discretized into $10 \times 10$ square pixels, so each pixel measures $1.5 \times 1.5 \mathrm{~mm}^{2}$. For simplicity, only one half of the antenna structure was modeled in the full-wave simulations by using a PMC symmetry setup. Therefore, the number of decision variables is 50 . The objective function to be minimized is the maximum $\left|S_{11}\right|$ over the sample frequency $F$, where $F=\{3,4.5,6,7.5,9,10.6 \mathrm{GHz}\}$.

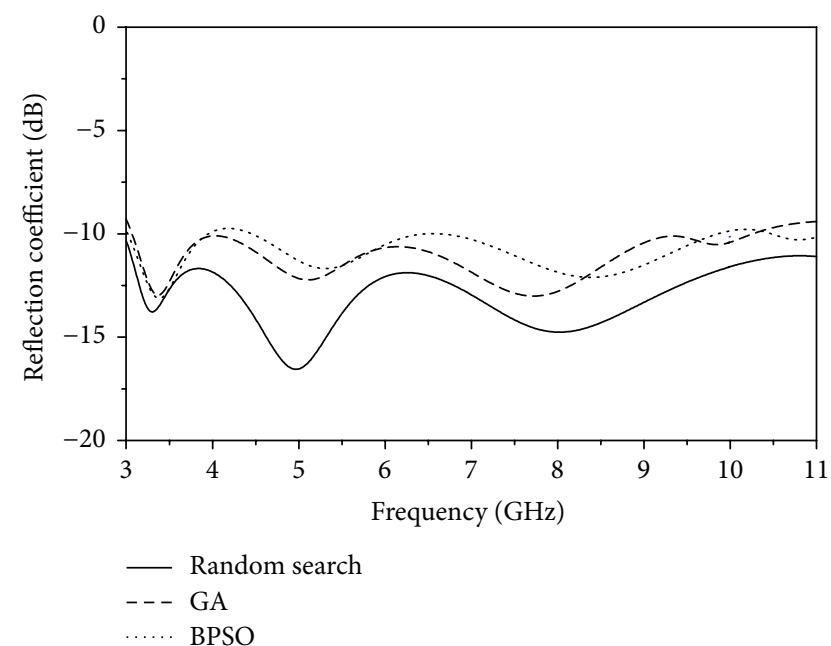

FIGURE 7: Simulated reflection coefficients of the optimized UWB monopole antennas corresponding to the GA, the BPSO, and the random search algorithm.

The resultant impedance matching of the three optimal structures is shown in Figure 7. Surprisingly, the performances of the random search algorithm are better than those of the GA and the BPSO. In particular, the GA found the optimum solution in the 25th iteration, the BPSO obtained the optimum structure in the 21st iteration, and the random search algorithm achieved the optimum performance at the 2,192nd look. Hence, GAs and BPSO do not dominate the random search algorithm in both the efficiency and solution quality. In addition, similar results are observed even though the parameterization is changed to other levels; in sum, the topology optimization problem is so difficult that GAs and BPSO are not more efficient than the random search algorithm.

2.4. Discussion. At this moment, it has been observed that binary optimization algorithms are not as effective as they should be. Such an unexpected result can be explained by the schema theorem [33]. Under proportionate selection and one-point crossover, the schema theorem can be written as follows:

$$
\begin{aligned}
& \langle m(H, t+1)\rangle \\
& \quad \geq m(H, t) \frac{f(H, t)}{\bar{f}(t)}\left(1-p_{c} \frac{\delta(H)}{l-1}-p_{m} \cdot o(H)\right),
\end{aligned}
$$

where $\langle m(H, t+1)\rangle$ is the expected number of individuals that belong to the schema $H$ at iteration $t+1, m(H, t)$ is the number of individuals that belong to the schema $H$ at iteration $t, f(H, t)$ is the average objective function value of the individuals belonging to $H$ at iteration $t, \bar{f}(t)$ is the average objective function value of the entire population at iteration $t, \delta(H)$ is the defining length, which represents the distance between the outermost defining positions of a schema, $l$ is the string length, and $o(H)$ is the order, 
representing the number of the defining positions of a schema.

From schema theorem (2), it is observed that only minimal, sequential, and superior schemata grow since $\delta(H)$ and $o(H)$ have minus signs. In other words, if the coadapted sets of alleles, namely, the building blocks, are widely separated, the crossover operator may often disrupt the schema because the cross-site tends to fall in between the outermost designing positions. On the other hand, if the building blocks in topology problem have too many defining positions, the mutation operator is inclined to disrupt the schema since the numerous defining positions are more difficult to survive intact. Consequently, the solution space cannot be efficiently explored unless the superior schemata grow. These statements have been verified by the earlier studies in the area of evolutionary computation (EC) [34, 35]. These papers have reported that only tight linkage (namely, smaller defining length) building blocks can be handled by heuristic algorithms; once the particularly important building blocks belong to loose linkage or random linkage, binary optimizers fail to find the optimum solution unless the population size is increased exponentially.

Unfortunately, topology optimization belongs to the class of problem having loose linkage building blocks. As shown in Figure 1, antennas are usually highly connected structures. This further means that numerous pixels should be considered together so that the physical structure will be unbroken; therefore, the corresponding alleles must coadapt to each other. However, when binary optimization algorithms encode the physical structure into one-dimensional (1D) bit string, the two-dimensional (2D) geometry is distorted because the neighboring information is lost. As a result, the structure of building blocks exhibits a loose linkage with higher-order defining positions and large defining lengths. Since the binary optimization algorithms fail to identify the building blocks, mostly, the operations by crossover and mutation disrupt superior schemata instead of making the best ones grow and taking over a dominant market share of the population. As a result, because of these broken schemata, the binary optimization algorithms have always suffered their poor convergence characteristic.

Although the deduction of problem difficulty is derived from a particular selection mechanism (proportionate selection) and a particular crossover procedure (one-point crossover), the generalization can be accomplished by rewriting (2) as

$$
\begin{aligned}
\langle m(H, t+1)\rangle \geq & m(H, t) \phi\left(H, m_{i}, f_{i}, t\right) \\
& \cdot P_{S}\left(H, m_{i}, f_{i}, t\right)\left(1-p_{m} \cdot o(H)\right),
\end{aligned}
$$

where $\varphi$ is a generalized selection taken as a function of the objective function $f_{i}$ and the distribution of structures in the population $m_{i}$ at iteration $t$ and $P_{S}$ is the survival probability under the crossover operators. In the previous cases, $\varphi\left(H, m_{i}, f_{i}, t\right)$ is the selection pressure $s=2$, and $P_{S}\left(H, m_{i}, f_{i}, t\right)$ can be written as $1-p_{c} \times \varepsilon$, where $\varepsilon$ is a bound on the disruption loss due to crossover. From such a generalization, it is observed that the speculation for the problem difficulty of topology optimization is still applicable.

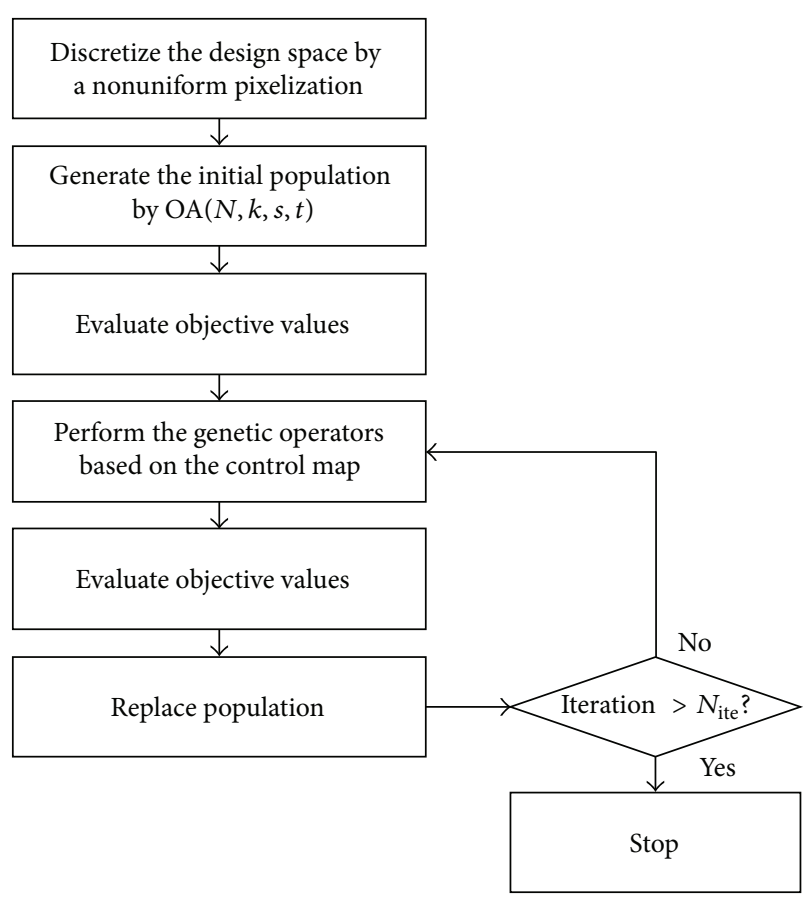

FIGURE 8: Flowchart of the building-block favoring method.

Hence, the defining lengths of the building blocks are so large that the resultant problem difficulty belongs to the class of deceptive trap functions, which are too difficult to be solved in reasonable time.

\section{The Proposed Building-Block Favoring Method}

With a full understanding on the problem difficulty of topology optimization, a new design technique aimed at applying topology optimization to internal antenna design is proposed. Figure 8 shows the flowchart of the proposed technique. This building-block favoring method is based on the framework of GAs, yet it uses a nonuniform discretization for the design space to tighten the defining positions of building blocks; in addition, the initial population is organized by orthogonal arrays (OAs) $[36,37]$ instead of randomized chromosomes so that sufficient diversity and numbers of building blocks are present initially. Furthermore, in order to ensure the growth of schemata, the control map of parameterization is carefully designed. The detailed procedure is as follows.

3.1. Nonuniform Discretization of a Design Space. Conventionally, the design space is discretized into uniform pixels, as shown in Figure 9(a). As discussed in the previous section, such a discretization results in broken building blocks with larger defining lengths and higher orders, so the resultant solution domain is very sensitive to design changes when the topology is close to optimum but insensitive to them when the topology is far from optimum. In addition, this intensive discretization requires a large number of pixels. These 


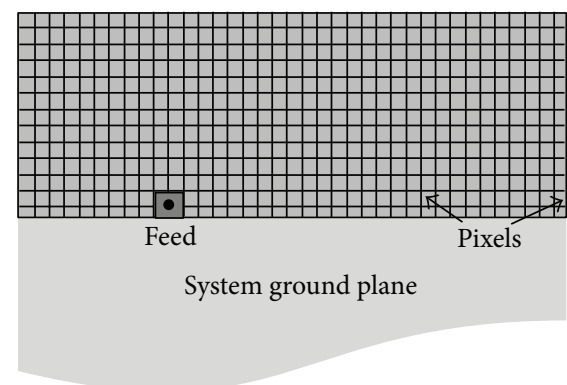

(a)

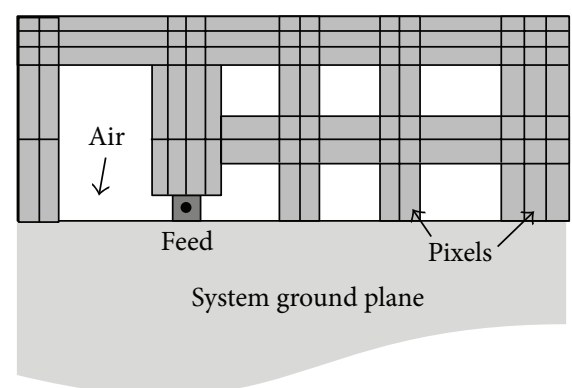

(b)

FIGURE 9: (a) Conventional discretization and (b) proposed nonuniform discretization of a design space.

limitations lead the uniform discretization to a completely intractable problem nature. Therefore, this paper proposes nonuniform discretizations incorporating prior knowledge or experience to reduce the problem difficulty. That is, based on the potential resonant current paths within the given area, the design space is roughly discretized into rectangular pixels with different sizes; for example, if a dual-band operation is desired, the solution domain can be roughly formed and subjected to the inverted-F topology with multiple current paths, as shown in Figure 9(b). As a result, the defining length of building blocks is significantly reduced, and the number of decision variables becomes much lesser, so the problem difficulty is relaxed into building-block-wise solvable. Some early reports have also used nonuniform pixels to enhance the optimized performances $[38,39]$. Although the associated performance of a nonuniform discretization will be meshdependent, it is a trade-off between human intervention and problem complexity. Once the technical knowledge is involved in the discretization, the robustness is enhanced, and the computational time is greatly reduced.

3.2. Initial Supply of Sufficient Building Blocks. After forming the solution space, the building-block favoring method proceeds to the optimization stage. However, in order to ensure the presence of the raw building blocks for genetic search, the initial population must have the best diversity, containing a larger number of fairly distributed building blocks so that the building blocks will be selected in the earlier stages of a run.

To equip the initial population with fairly and uniformly distributed candidates, the proposed method uses OAs as the initial population. By definition, an $\mathrm{OA}(N, k, s, t)$ is an $N \times k$ matrix, each element of which has only $s$ distinct level values, and every $N \times t$ submatrix of the OA contains each $t$-tuple exactly $N / s^{t}$ times. When $\mathrm{OA}(N, k, s, t)$ is applied to the arrangement of initial population, the parameter $s$ is regarded as the number of states of decision variable, so $s$ is equal to 2 in topology optimization problems. In addition, $k$ is the number of pixels, and $N$ is the population size. If the strength $t$ is chosen as 2 , for each pixel, levels 0 (air) and 1 (conductor) occur equally $N / 2$ times in all $N$ populations, thereby achieving a balanced distribution for the initial population. The following are two examples of OAs:

$$
\begin{aligned}
\mathrm{OA}(4,3,2,2) & =\left[\begin{array}{lll}
0 & 0 & 0 \\
0 & 1 & 1 \\
1 & 0 & 1 \\
1 & 1 & 0
\end{array}\right], \\
\mathrm{OA}(8,4,2,3) & =\left[\begin{array}{llll}
0 & 0 & 0 & 0 \\
0 & 0 & 1 & 1 \\
0 & 1 & 0 & 1 \\
0 & 1 & 1 & 0 \\
1 & 0 & 0 & 1 \\
1 & 0 & 1 & 0 \\
1 & 1 & 0 & 0 \\
1 & 1 & 1 & 1
\end{array}\right],
\end{aligned}
$$

where $\mathrm{OA}(4,3,2,2)$ is suitable for a topology optimization problem with three candidate pixels. In this case, four chromosomes are used, and their initial states are assigned as $(0,0,0),(0,1,1),(1,0,1)$, and $(1,1,0)$. Similarly, $\mathrm{OA}(8,4,2,3)$ is suitable for a topology optimization problem with four candidate pixels, and the initial population can be assigned as $(0,0,0,0),(0,0,1,1),(0,1,0,1),(0,1,1,0)$, $(1,0,0,1),(1,0,1,0),(1,1,0,0)$, and $(1,1,1,1)$. It can be seen that these candidate solutions are scattered evenly in the solution space; therefore, the proposed method can explore the entire solution space with equal density. As the lowerorder building blocks converge, the other candidate solution may move closer to the global optimum. On the other hand, OAs have another useful feature: swapping or deleting the columns does not alter the orthogonality property of the array. This property facilitates the construction of an $\mathrm{OA}$ database for arbitrary number of pixels $k$. If an OA for certain $k$ is desired, it can be obtained simply by discarding the excessive columns from a known OA with larger $k$.

For verification purpose, the OA initialization is tested for its convergence characteristic. The instance to be tested 


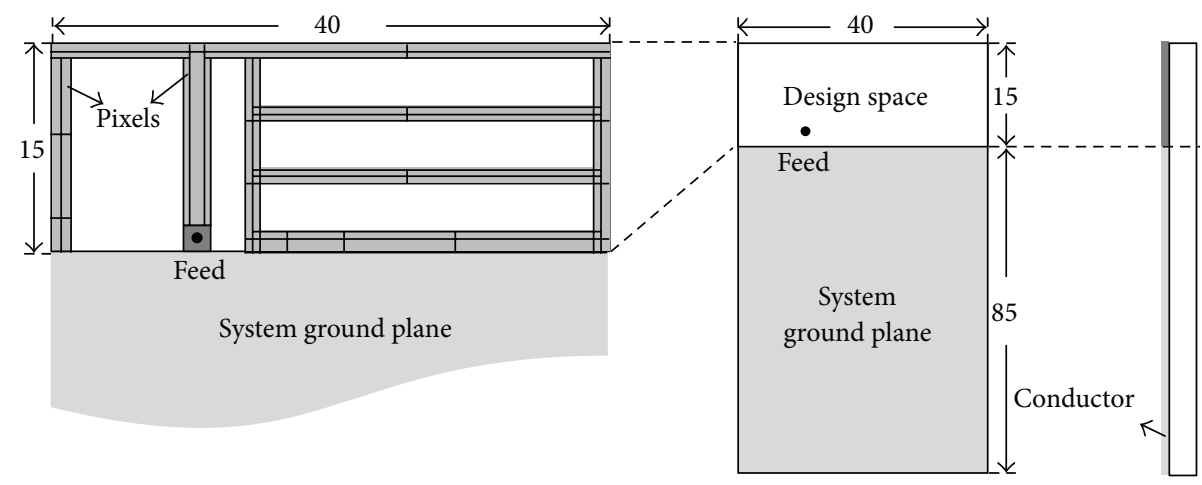

FIGURE 10: Geometry of the mobile antenna for handheld devices and the discretization of the design space (unit: $\mathrm{mm}$ ).

is given by a mobile phone application, the physical environment of which is shown in Figure 10. The entire circuitry is fabricated on a $0.8 \mathrm{~mm}$ thick FR4 substrate with an area of $40 \times 100 \mathrm{~mm}^{2}$. A ground plane of length $85 \mathrm{~mm}$ and width $40 \mathrm{~mm}$ is printed on the top of the circuit board, and an unoccupied area of $40 \times 15 \mathrm{~mm}^{2}$ is left as a design space available for the pixelated antenna design. The antenna is fed by a 50 -ohm coaxial cable. The solution domain is roughly formed into 57 nonuniform pixels based on the common inverted-F topology. The objective function to be minimized is the magnitude of the reflection coefficient at $900 \mathrm{MHz}$.

In this case, the $\mathrm{OA}(64,63,2,2)$ is suitable for generating uniformly distributed building blocks. 57 out of 63 columns are randomly selected and assigned to each individual. Also, the population size is assigned as $N_{\text {pop }}=64$. Hence, each pixel has $64 / 2=32$ times of occurrences as 1 (metal) and 32 times of occurrences as 0 (air) in the initial population. The strength $t=2$ further illustrates that each combination of arbitrary two pixels, namely, $(1,1),(1,0),(0,0)$, and $(0,1)$, exists $64 / 2^{2}=16$ times of occurrences, respectively. To compare the efficacy between the OA initialization and random initialization, these two initialization manners are tested for 10 repeating trials, respectively. In each trial, they use the same size of population $\left(N_{\text {pop }}=64\right)$, performing the same number of functional evaluations. The other parameterization is fixed as the number of iterations $N_{\text {ite }}=40$, the number of preserved elitists $N_{\text {elit }}=4$, and selection pressure for tournament selection without replacement $s=2$, and probabilities for uniform crossover $p_{c}$ and mutation $p_{m}$ are 0.5 and 0.01 , respectively. After recording the convergence history, namely, the best and average fitness value versus iteration for each trial, the average convergence histories for the 10 trials are exhibited in Figure 11. Clearly, the convergence history of average fitness value shows that the OA initialization has a faster convergence, and the best objective value determined by the $\mathrm{OA}$ is superior to that obtained via the randomized initialization. These results verify that OAs can supply fairly distributed building blocks, and the OA initialization leads to a better convergence characteristic.

3.3. Control Map of the Proposed Method. After generating a uniformly scattered initial population, the proposed method iterates and improves the population of candidate solutions. This optimization process is based on the genetic operators, namely, selection, crossover, mutation, and elitism replacement; however, the inclination of the algorithm must be carefully designed so that the complex building blocks can converge. It is known that two main features must be balanced in constructing a successful optimization: the degree of exploitation and the degree of exploration. Such features are controlled by the parameterization of selection pressure $s$, crossover probability $p_{c}$, and mutation probability $p_{m}$.

The parameterization must ensure the growth of schemata. Based on generalized schema theorem (3), the growth of schemata requires $\varphi\left(H, m_{i}, f_{i}, t\right) \times P_{S}\left(H, m_{i}, f_{i}, t\right) \geq$ 1 . For the topology optimization problem with tournament section and uniform crossover, this is equivalent to

$$
s\left[1-p_{c} \varepsilon\right] \geq 1 \text {. }
$$

Considering the worst-case scenario, namely, when the loss of schema integrity is total (when $\varepsilon=1$ ), the limiting $p_{c}$ value ensuring the building-block market share grows is

$$
p_{c} \leq 1-\frac{1}{s}
$$

as (6) can be understood as the control map of topology optimization problems. Usually, the selection pressure is chosen as 2 to prevent the population from premature convergence, so $p_{c}$ should not be larger than 0.5 .

In order to verify the proposed control map, $p_{c}$ is tested for three different levels: $p_{c}=0.3, p_{c}=0.5$, and $p_{c}=0.8$. The design scenario is again the mobile antenna for handheld devices at $900 \mathrm{MHz}$, shown in Figure 10. Each level is tested for 10 repeating trials. The other parameterization is fixed as $N_{\text {ite }}=40, N_{\text {elit }}=4, s=2$, and $p_{m}=0.01$. The best objective values and the average objective values among the 64 chromosomes versus iteration are recorded, and the average results for these 10 trials are shown in Figure 12. Clearly, $p_{c}=0.3$ and $p_{c}=0.5$ yield superior objective values, and they also have a more desired convergence characteristic. In contrast, a larger crossover probability, namely, $p_{c}=0.8$, spends relatively too much effort exploring distant regions; the building blocks do not converge, so the best objective value is worse than the other two levels. 


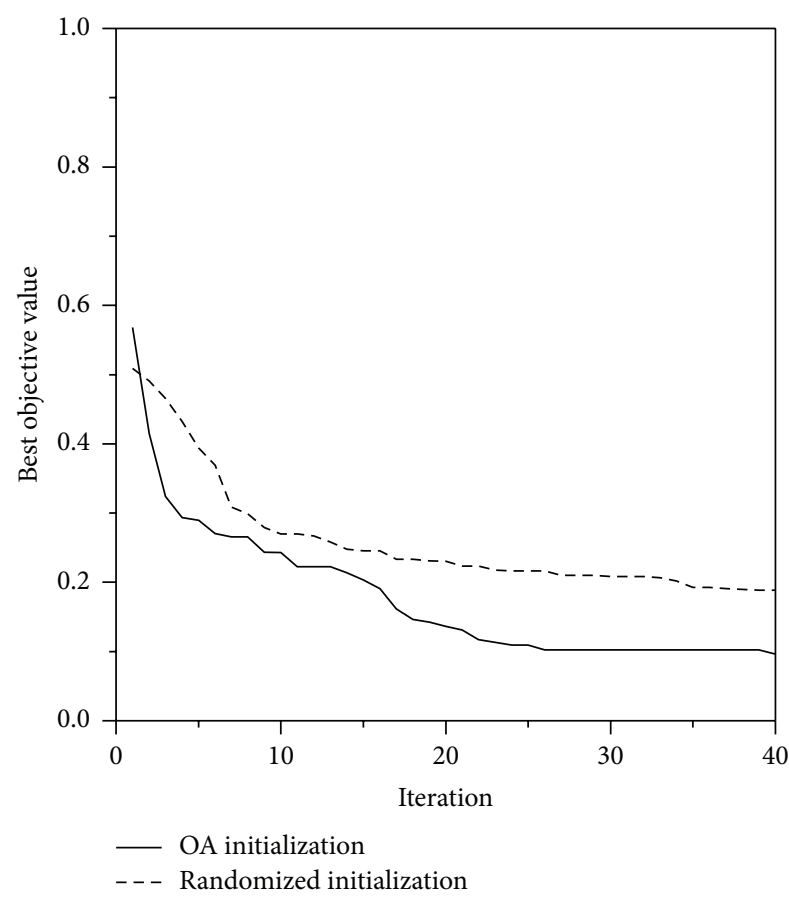

(a)

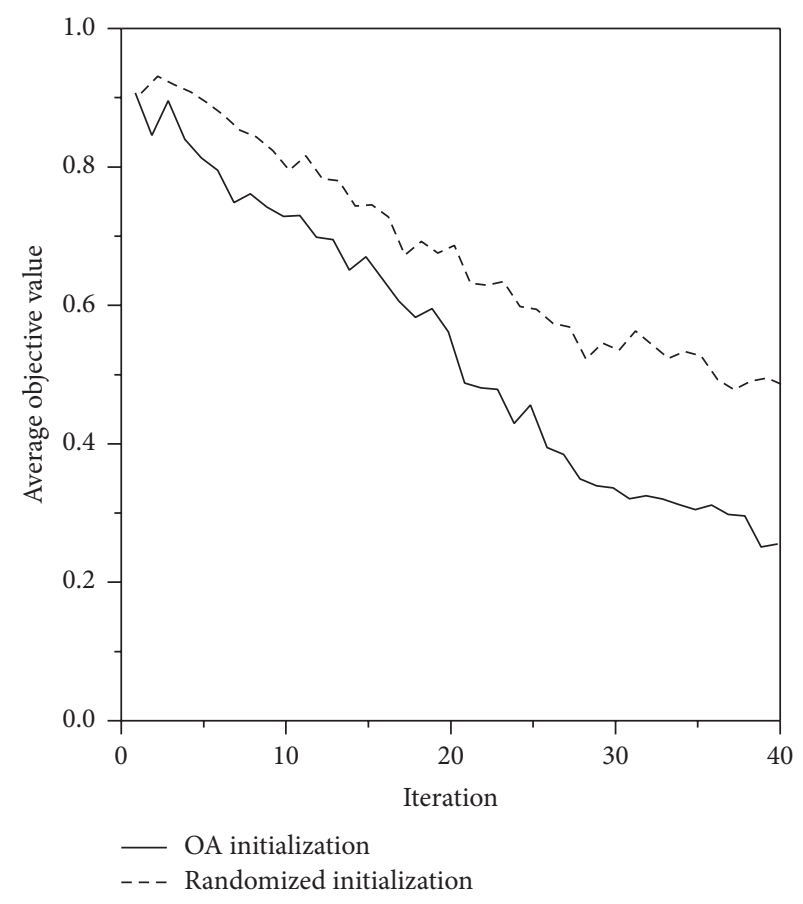

(b)

FIGURE 11: Convergence histories of (a) best objective value and (b) average objective value for the randomized initialization and the OA initialization.

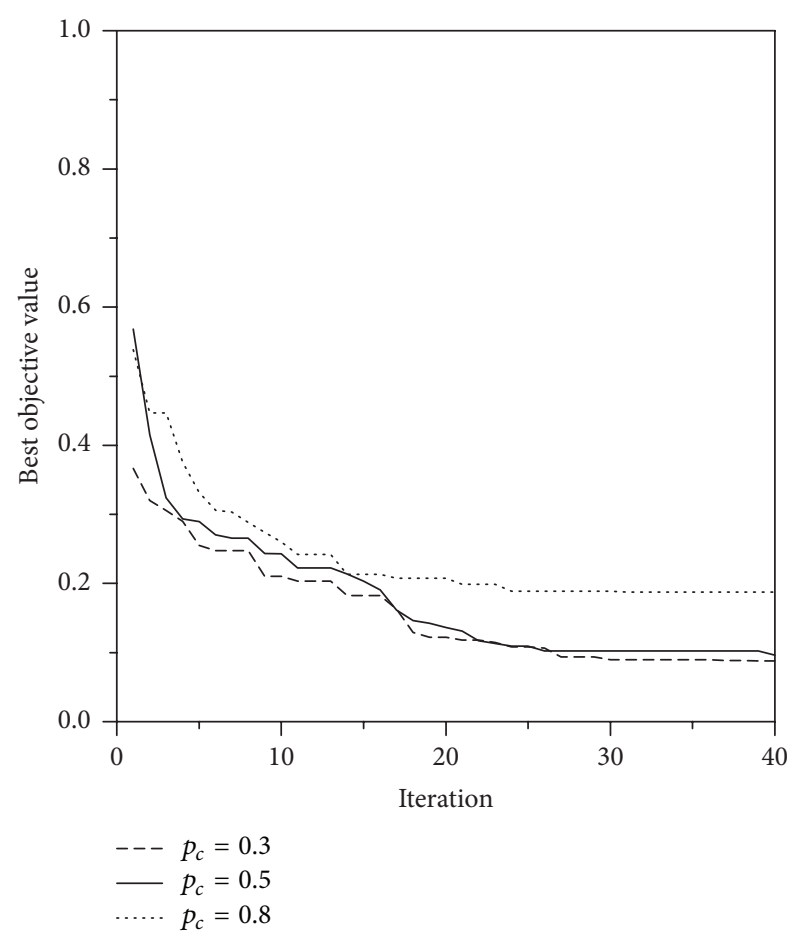

(a)

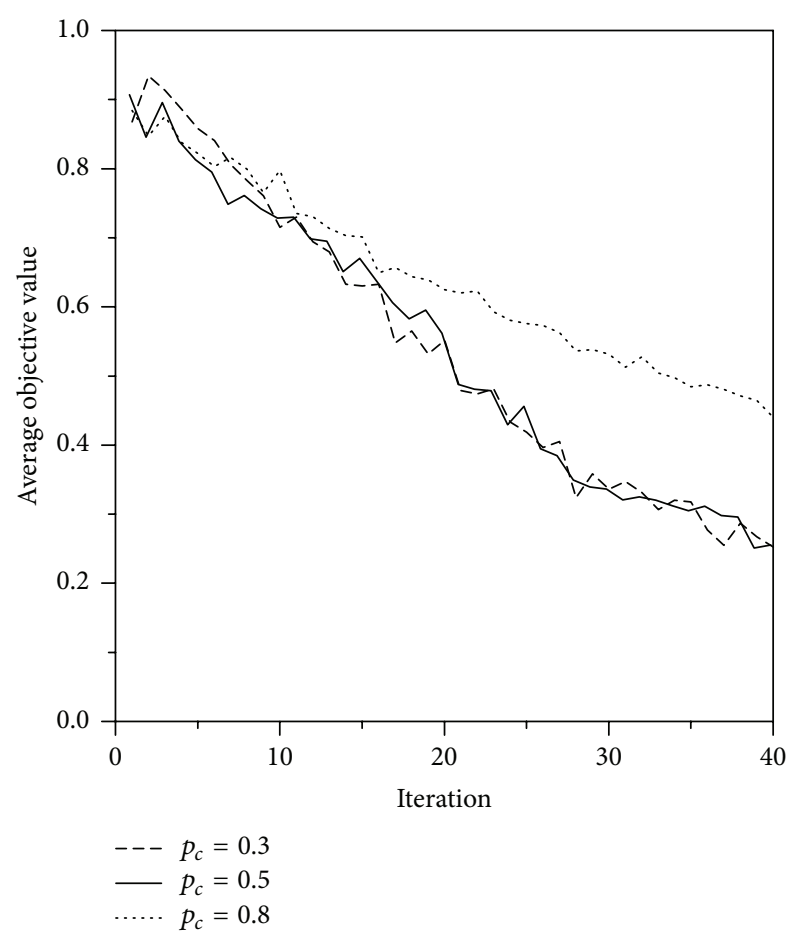

(b)

FIGURE 12: Convergence histories of (a) best objective value and (b) average objective value for $p_{c}=0.3, p_{c}=0.5$, and $p_{c}=0.8$. 
In sum, the proposed control map facilitates the necessary exchange of intact building blocks; meanwhile, low-order building blocks will be selected in the early iterations, thereby permitting the assembly of the complex building blocks needed to solve topology optimization problems.

\section{Internal Antenna Design}

Next, the capability of the proposed building-block favoring method is examined by two design problems. Since topology optimization is particularly suitable for internal antenna design, its efficacy is verified by a mobile antenna for handheld devices and an internal antenna for laptop applications. To validate the proposed approach, the antenna designs are also conducted by the conventional topology optimization, which uses a number of uniform pixels and a randomized population for initialization.

4.1. A Mobile Antenna for Handheld Devices. The first design problem is an internal antenna for handheld devices, the environment of which has been shown in Figure 10. The design goal is to generate a dual-band operation covering 824$960 \mathrm{MHz}$ and $1710-2170 \mathrm{MHz}$; therefore, the objective function can be assigned as a minimization of the sum of $\left|S_{11}\right|^{3}$, $j=\{820,880,900,960,1710,1940,2170 \mathrm{MHz}\}$. In this case, the initial population is created by the $\mathrm{OA}(64,63,2,2)$, so the population size is 64. The other parameterization is $N_{\text {ite }}=$ $40, N_{\text {elit }}=4, s=2, p_{c}=0.4$, and $p_{m}=0.01$. On the other hand, this problem is also handled by the conventional topology optimization; that is, the rectangular design space is discretized into $20 \times 8$ pixels, and each pixel measures $2 \times$ $1.875 \mathrm{~mm}^{2}$. The objective function is the same as the previous case, and the parameterization is also fixed at the same level except for the setup of initialization, which is a randomized population comprising 64 chromosomes instead of the OA. In order to fairly determine which method is indeed better, the same number of functional evaluations is used. That is, the total number of functional calls is set to 2,560 no matter what optimization approach is tested.

The optimized performances of the proposed method and the conventional method are shown in Figure 13. It can be seen that the proposed method generates a dual-band operation, while the conventional method fails to find a desired performance. The total computational time is about 30 hours, determined mainly by the 2,560 full-wave simulations. Clearly, the proposed method automatically achieves a good antenna design, fulfilling the requirement of a lower development cost and a shorter design cycle.

For verification purpose, the optimized antenna determined by the proposed method is also fabricated and tested. The measured reflection coefficients are also exhibited in Figure 13. These results confirm that the optimized antenna covers the lower band $(824-960 \mathrm{MHz})$ and higher band $(1710-2170 \mathrm{MHz})$ by $3: 1$ VSWR, which is widely used as the specification of internal antennas. Besides, the antenna efficiencies with mismatching loss included are shown in Figure 14. The efficiencies are greater than $45 \%$. These results

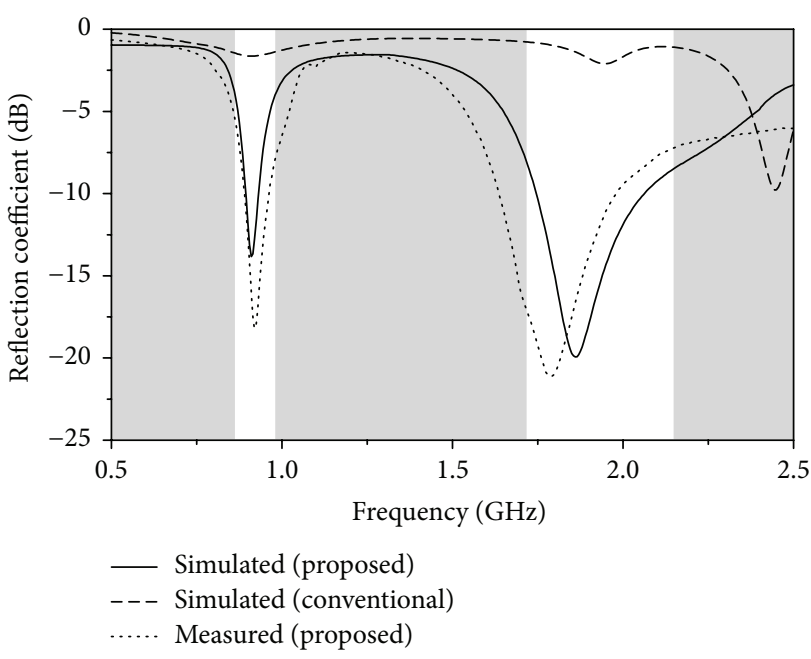

FIGURE 13: Simulated and measured reflection coefficients of the optimized mobile antenna. The simulated performances for the conventional topology optimization are also provided.

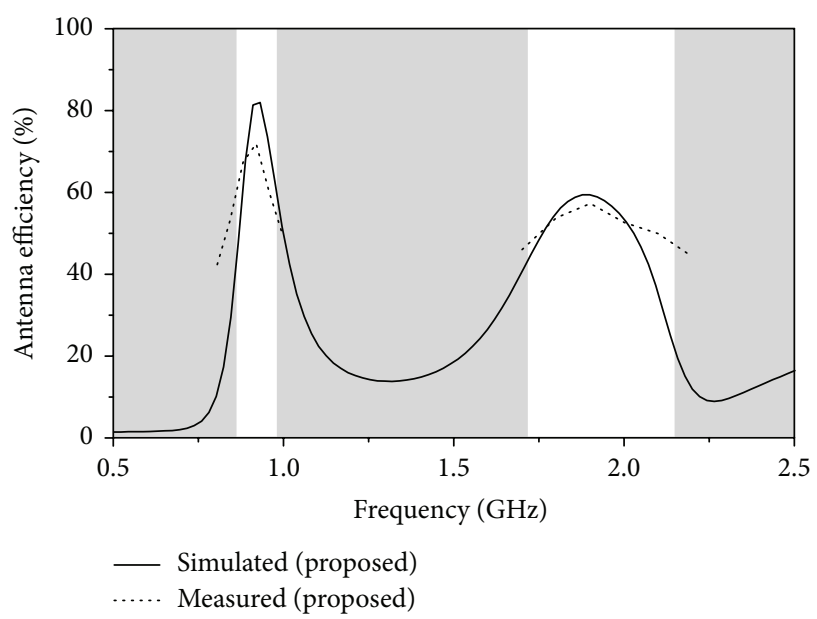

Figure 14: Simulated and measured antenna efficiencies of the optimized mobile antenna (with mismatching loss included).

indicate that the optimized antenna is suitable for wireless communication in handheld devices.

4.2. An Internal Antenna for Laptop Applications. The second design problem is an internal antenna for laptop applications. The design environment is shown in Figure 15. The area of the design space is $8 \times 60 \mathrm{~mm}^{2}$, and this design space is discretized into 53 pixels, which is roughly formed based on the inverted-F topology with multiple current paths. The antenna is designed on a FR4 substrate of thickness $0.8 \mathrm{~mm}$. The design goal is again a dual-band operation including $824-960 \mathrm{MHz}$ and $1710-2170 \mathrm{MHz}$, so the objective to be minimized is the sum of $\left|S_{11}\right|^{3}, j=$ $\{820,880,900,960,1710,1940,2170 \mathrm{MHz}\}$.

The proposed technique is applied to this problem to optimize the antenna performances. In particular, 


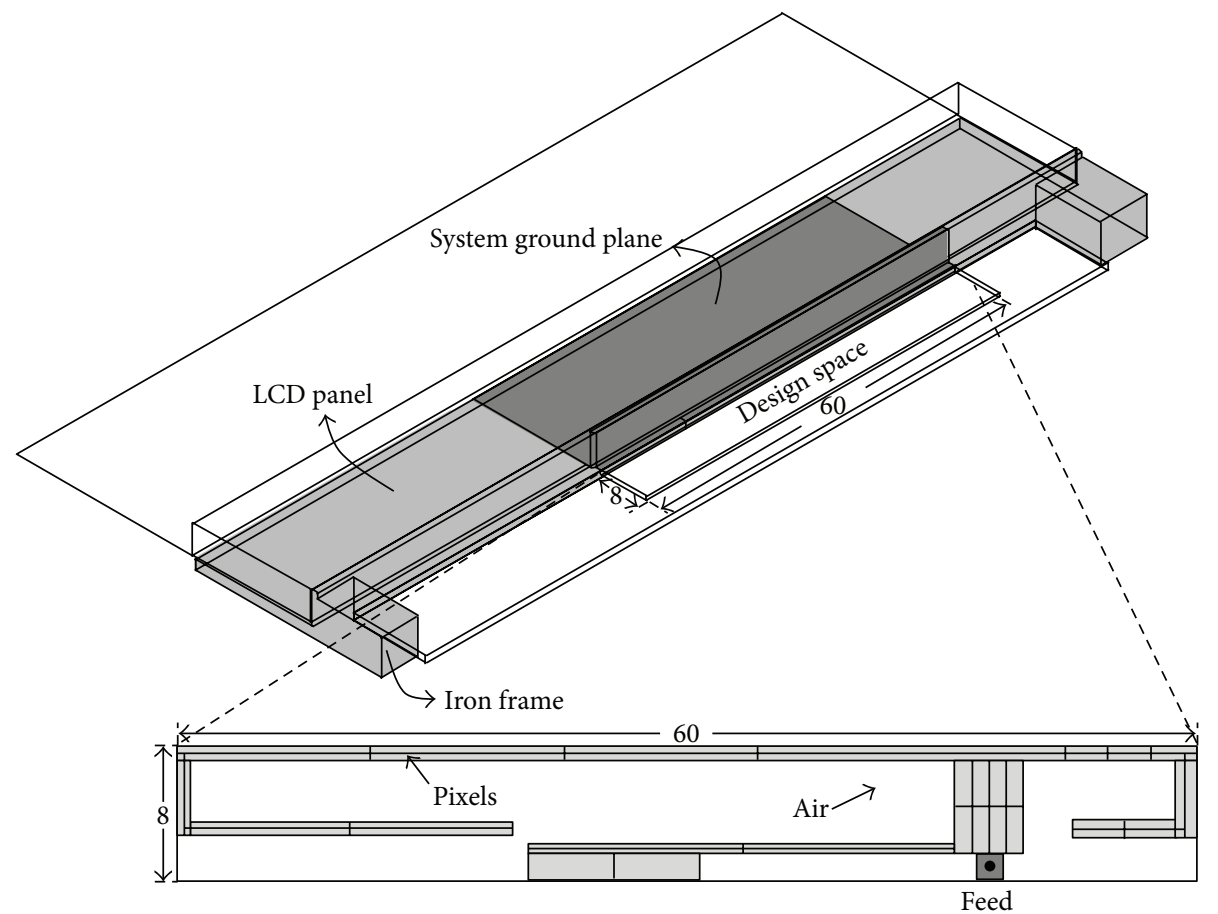

FIGURE 15: Geometry of the internal antenna for laptop applications and the discretization of the design space (unit: mm).

$\mathrm{OA}(64,63,2,2)$ is assigned as the initial population, and other parameters are $N_{\text {ite }}=40, N_{\text {elit }}=4, s=2, p_{c}=0.4$, and $p_{m}=0.01$. Additionally, the conventional topology optimization with uniform pixelization is also applied to this scenario; the design space is discretized into $4 \times 20$ pixels, and each pixel measures $2 \times 2 \mathrm{~mm}^{2}$. With the same objective function and parameterization except that the initialization is a randomized population, the conventional topology optimization finds another optimum structure after 2,560 tests.

The resultant reflection coefficients determined by the proposed technique and those obtained by the conventional topology optimization are shown in Figure 16. Clearly, only the building-block favoring method yields desired performances, and the conventional method cannot attain a dualband operation. Furthermore, the optimized antenna determined by the proposed technique is also fabricated and tested, and the measured reflection coefficients are shown in Figure 16. They exhibit very good agreement. These results confirm that the building-block favoring method is very suitable for internal antenna design.

\section{Conclusion}

This paper has proposed a novel design technique to favor the building blocks in the topology optimization problems. Analysis of the importance of the building blocks has been considered, and the numerical results have shown that the conventional topology optimization constructs loose linkage building blocks with large defining lengths and high orders; hence, the problem difficulty is so complex that no

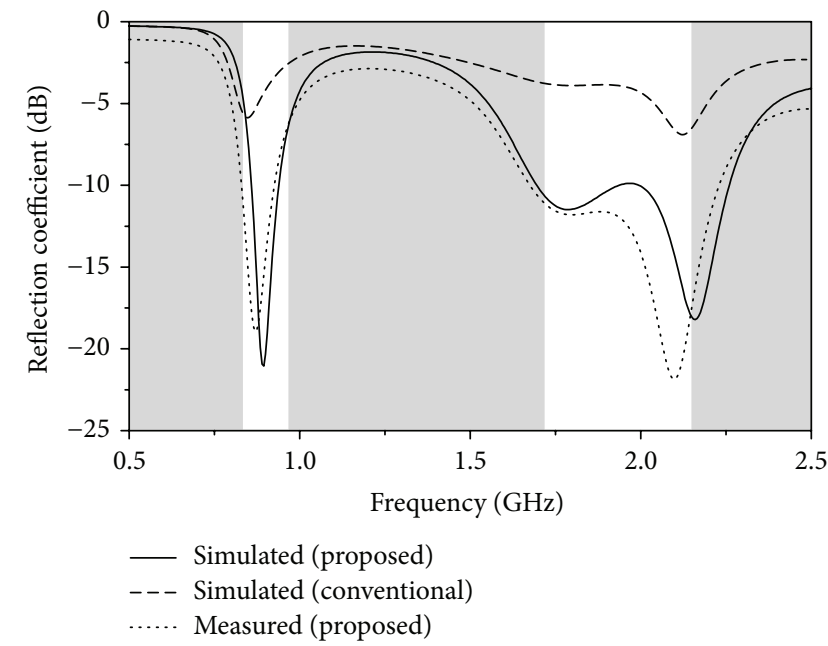

FIGURE 16: Simulated and measured reflection coefficients of the optimized internal antenna. The simulated performances for the conventional topology optimization are also provided.

binary optimization algorithms are more efficient than the random search algorithm. According to what the problem really needs, the proposed method aims to enhance the efficiency and robustness of topology optimization, which is significantly improved by three mechanisms. Firstly, the proposed method uses a nonuniform discretization, which makes hard problems easy by forming tight linkage groups of building blocks. Secondly, the initial population is organized by OAs, which ensure adequate supply of the raw 
building blocks in a population. Lastly, the parameterization is constructed by ensuring the growth of schemata. As a result, the proposed technique successfully develops two dual-band internal antennas covering $824-960 \mathrm{MHz}$ and $1710-2170 \mathrm{MHz}$. The simulated and measured results show that the performances of the building-block favoring method are much better than those determined by the conventional topology optimization. In addition to these design instances, the proposed technique is generally applicable to other design problems of internal antennas, thereby leading to a shorter design cycle and a lower development cost.

\section{Conflict of Interests}

The author declares that there is no conflict of interests regarding the publication of this paper.

\section{Acknowledgment}

This work was supported by the Ministry of Science and Technology, Taiwan, under Contracts MOST 103-2221-E-027004 .

\section{References}

[1] N. Tokan, "Optimization of the UWB feed antenna position in reflector applications," International Journal of Antennas and Propagation, vol. 2014, Article ID 961818, 7 pages, 2014.

[2] P. J. Williams and T. C. Molteno, "A comparison of genetic programming with genetic algorithms for wire antenna design," International Journal of Antennas and Propagation, vol. 2008, Article ID 197849, 6 pages, 2008.

[3] M. Zubair and M. Moinuddin, "Joint optimization of microstrip patch antennas using particle swarm optimization for UWB systems," International Journal of Antennas and Propagation, vol. 2013, Article ID 649049, 8 pages, 2013.

[4] C. Yu, T. Xu, and C. Liu, "Design of a novel UWB omnidirectional antenna using particle swarm optimization," International Journal of Antennas and Propagation, vol. 2015, Article ID 303195, 7 pages, 2015.

[5] S. Koziel, J. Meng, J. W. Bandler, M. H. Bakr, and Q. S. Cheng, "Accelerated microwave design optimization with tuning space mapping," IEEE Transactions on Microwave Theory and Techniques, vol. 57, no. 2, pp. 383-394, 2009.

[6] Z. Wang and S. Fang, "ANN synthesis model of single-feed corner-truncated circularly polarized microstrip antenna with an air gap for wideband applications," International Journal of Antennas and Propagation, vol. 2014, Article ID 392843, 2014.

[7] M. P. Bendsøe and N. Kikuchi, "Generating optimal topologies in structural design using a homogenization method," Computer Methods in Applied Mechanics and Engineering, vol. 71, no. 2, pp. 197-224, 1988.

[8] D. N. Dyck and D. A. Lowther, "Automated design of magnetic devices by optimizing material distribution," IEEE Transactions on Magnetics, vol. 32, no. 3, pp. 1188-1192, 1996.

[9] C. Delabie, M. Villegas, and O. Picon, "Creation of new shapes for resonant microstrip structures by means of genetic algorithms," Electronics Letters, vol. 33, no. 18, pp. 1509-1510, 1997.
[10] L. Alatan, M. I. Aksum, K. Leblebicioglu, and M. T. Birand, "Use of computationally efficient method of moments in the optimization of printed antennas," IEEE Transactions on Antennas and Propagation, vol. 47, no. 4, pp. 725-732, 1999.

[11] J. M. Michael Johnson and Y. Rahmat-Samii, "Genetic algorithms and method of moments (GA/MOM) for the design of integrated antennas," IEEE Transactions on Antennas and Propagation, vol. 47, no. 10, pp. 1606-1614, 1999.

[12] H. Choo, A. Hutani, L. C. Trintinalia, and H. Ling, "Shape optimization of broadband microstrip antennas using genetic algorithm," Electronics Letters, vol. 36, no. 25, pp. 2057-2058, 2000.

[13] D. S. Weile and E. Michielssen, "The use of domain decomposition genetic algorithms exploiting model reduction for the design of frequency selective surfaces," Computer Methods in Applied Mechanics and Engineering, vol. 186, no. 2-4, pp. 439$458,2000$.

[14] H. Choo and H. Ling, "Design of multiband microstrip antennas using a genetic algorithm," IEEE Microwave and Wireless Components Letters, vol. 12, no. 9, pp. 345-347, 2002.

[15] N. Herscovici, M. F. Osorio, and C. Peixeiro, "Miniaturization of rectangular microstrip patches using genetic algorithms," IEEE Antennas and Wireless Propagation Letters, vol. 1, pp. 94-97, 2002.

[16] Z. Li, Y. E. Erdemli, J. L. Volakis, and P. Y. Papalambros, "Design optimization of conformal antennas by integrating stochastic algorithms with the hybrid finite-element method," IEEE Transactions on Antennas and Propagation, vol. 50, no. 5, pp. 676-684, 2002.

[17] F. J. Villegas, T. Cwik, Y. Rahmat-Samii, and M. Manteghi, "A parallel electromagnetic genetic-algorithm optimization (EGO) application for patch antenna design," IEEE Transactions on Antennas and Propagation, vol. 52, no. 9, pp. 2424-2435, 2004.

[18] P. Soontornpipit, C. M. Furse, and Y. C. Chung, "Miniaturized biocompatible microstrip antenna using genetic algorithm," IEEE Transactions on Antennas and Propagation, vol. 53, no. 6, pp. 1939-1945, 2005.

[19] L. A. Griffiths, C. Furse, and Y. C. Chung, "Broadband and multiband antenna design using the genetic algorithm to create amorphous shapes using ellipses," IEEE Transactions on Antennas and Propagation, vol. 54, no. 10, pp. 2776-2782, 2006.

[20] S. Koulouridis, D. Psychoudakis, and J. L. Volakis, "Multiobjective optimal antenna design based on volumetric material optimization," IEEE Transactions on Antennas and Propagation, vol. 55, no. 3 I, pp. 594-603, 2007.

[21] A. Modiri and K. Kiasaleh, "Efficient design of microstrip antennas for SDR applications using modified PSO algorithm," IEEE Transactions on Magnetics, vol. 47, no. 5, pp. 1278-1281, 2011.

[22] E. Hassan, E. Wadbro, and M. Berggren, “Topology optimization of metallic antennas," IEEE Transactions on Antennas and Propagation, vol. 62, no. 5, pp. 2488-2500, 2014.

[23] A. J. Kerkhoff, R. L. Rogers, and H. Ling, "Design and analysis of planar monopole antennas using a genetic algorithm approach," IEEE Transactions on Antennas and Propagation, vol. 52, no. 10, pp. 2709-2718, 2004.

[24] A. J. Kerkhoff and H. Ling, "Design of a band-notched planar nonopole antenna using genetic algorithm optimization," IEEE Transactions on Antennas and Propagation, vol. 55, no. 3, pp. 604-610, 2007. 
[25] M. John and M. J. Ammann, "Wideband printed monopole design using a genetic algorithm," IEEE Antennas and Wireless Propagation Letters, vol. 6, pp. 447-449, 2007.

[26] M. Ding, R. Jin, and J. Geng, "Optimal design of ultra wideband antennas using a mixed model of 2-D genetic algorithm and finite-difference time-domain," Microwave and Optical Technology Letters, vol. 49, no. 12, pp. 3177-3180, 2007.

[27] M. Ding, R. Jin, J. Geng, Q. Wu, and G. Yang, "Auto-design of band-notched UWB antennas using mixed model of 2D GA and FDTD," Electronics Letters, vol. 44, no. 4, pp. 257-258, 2008.

[28] N. Jin and Y. Rahmat-Samii, "Hybrid real-binary particle swarm optimization (HPSO) in engineering electromagnetics," IEEE Transactions on Antennas and Propagation, vol. 58, no. 12, pp. 3786-3794, 2010.

[29] C.-F. Huang and H.-M. Li, "Design optimization of chip antennas using the GA-FDTD approach," International Journal of RF and Microwave Computer-Aided Engineering, vol. 15, no. 1, pp. 116-127, 2005.

[30] J. M. Jayasinghe, W. Jayasinghe, and D. Uduwawala, "A novel multiband miniature planar inverted $\mathrm{F}$ antenna design for Bluetooth and WLAN applications," International Journal of Antennas and Propagation, vol. 2015, Article ID 970152, 6 pages, 2015.

[31] N. Jin and Y. Rahmat-Samii, "Particle swarm optimization for multi-band handset antenna designs: a hybrid real-binary implementation," in Proceedings of the IEEE International Symposium on Antennas and Propagation, San Diego, Calif, USA, July 2008.

[32] J. M. J. W. Jayasinghe, J. Anguera, and D. N. Uduwawala, “A simple design of multi band microstrip patch antennas robust to fabrication tolerances for GSM, UMTS, LTE, and Bluetooth applications by using genetic algorithm optimization," Progress In Electromagnetics Research M, vol. 27, pp. 255-269, 2012.

[33] J. H. Holland, Adaptation in Natural and Artificial Systems, University of Michigan Press, Ann Arbor, Mich, USA, 1975.

[34] D. E. Goldberg, B. Korb, and K. Deb, "Messy genetic algorithms: motivation, analysis, and first results," Complex Systems, vol. 3, no. 5, pp. 493-530, 1989.

[35] G. Harik, Linkage Learning via Probabilistic Modeling in the ECGA, University of Illinois Press, Champaign, Ill, USA, 1999.

[36] A. S. Hedayat, N. J. A. Sloane, and J. Stufken, Orthogonal Arrays: Theory and Applications, Springer, New York, NY, USA, 1999.

[37] Y.-W. Leung and Y. Wang, "An orthogonal genetic algorithm with quantization for global numerical optimization," IEEE Transactions on Evolutionary Computation, vol. 5, no. 1, pp. 4153, 2001.

[38] J. M. J. W. Jayasinghe, J. Anguera, D. N. Uduwawala, and A. Andújar, "Nonuniform overlapping method in designing microstrip patch antennas using genetic algorithm optimization," International Journal of Antennas and Propagation, vol. 2015, Article ID 805820, 8 pages, 2015.

[39] A. E. Teirab, J. A. Jervase, and S. S. Mneina, "Design of UWB monopole antenna using genetic algorithms," in Proceedings of the 7th IEEE GCC Conference and Exhibition (GCC '13), pp. 8992, Doha, Qatar, November 2013. 

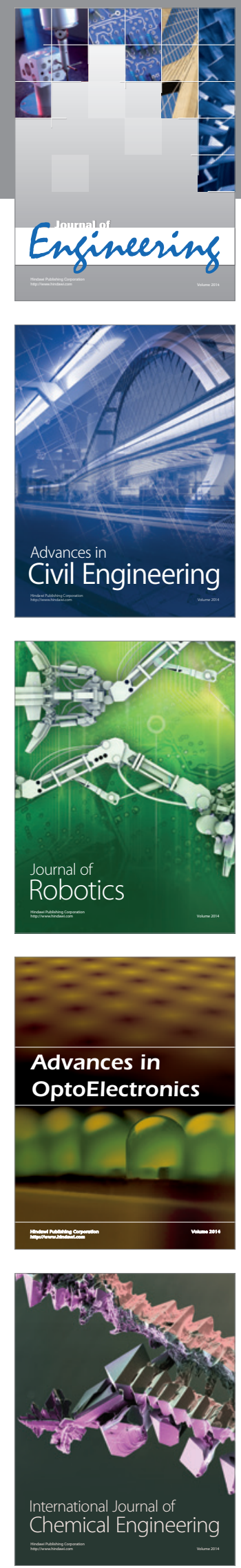

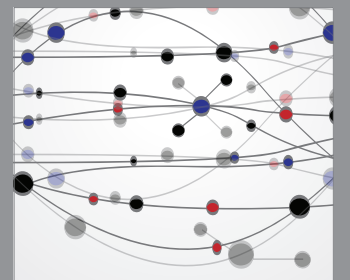

The Scientific World Journal
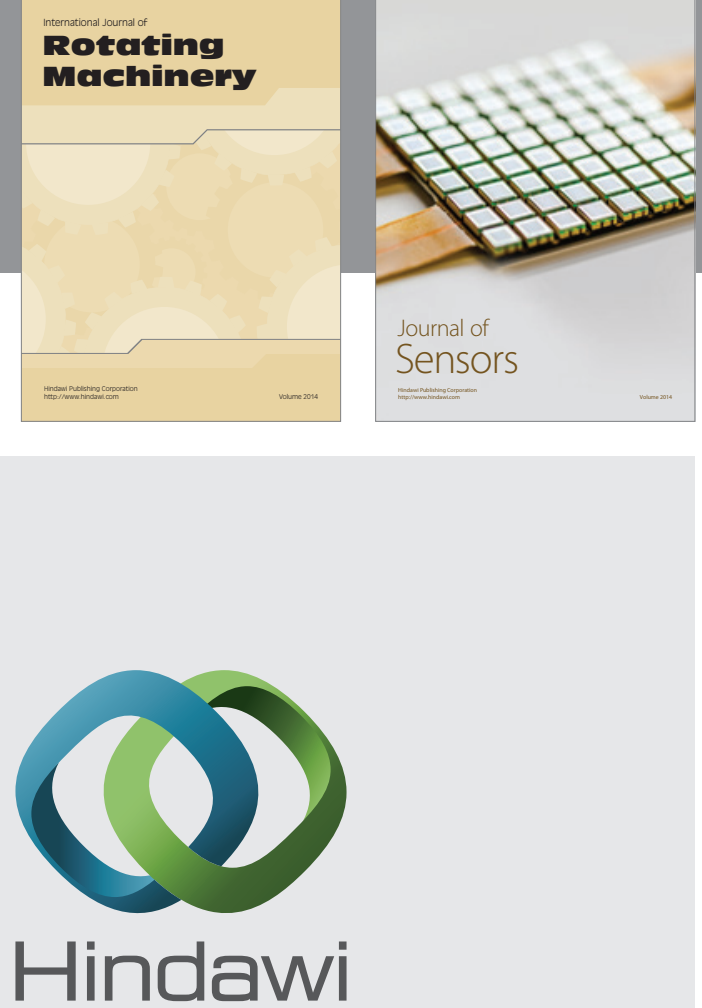

Submit your manuscripts at http://www.hindawi.com
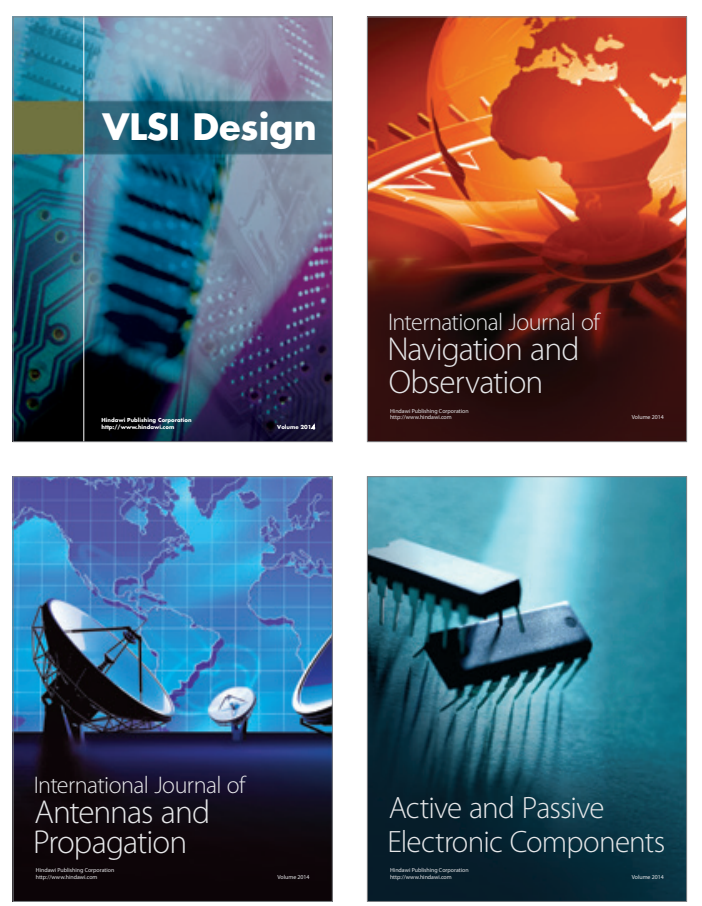
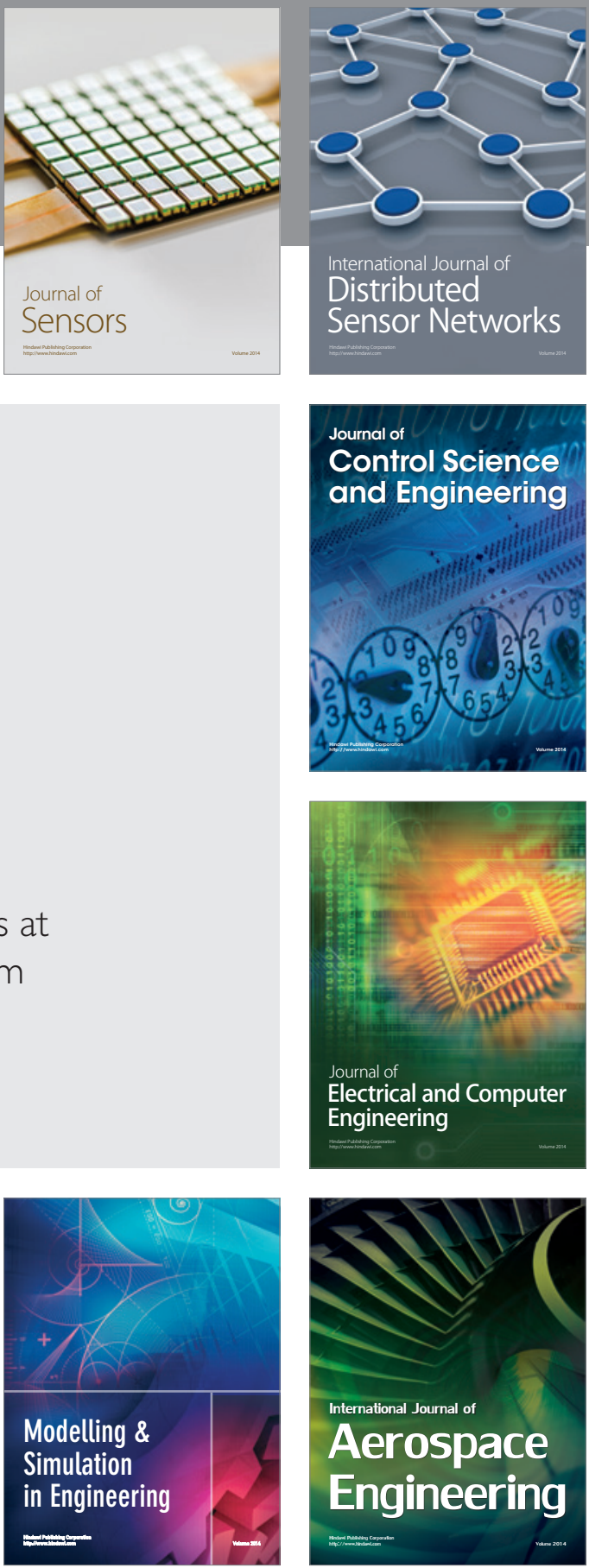

Journal of

Control Science

and Engineering
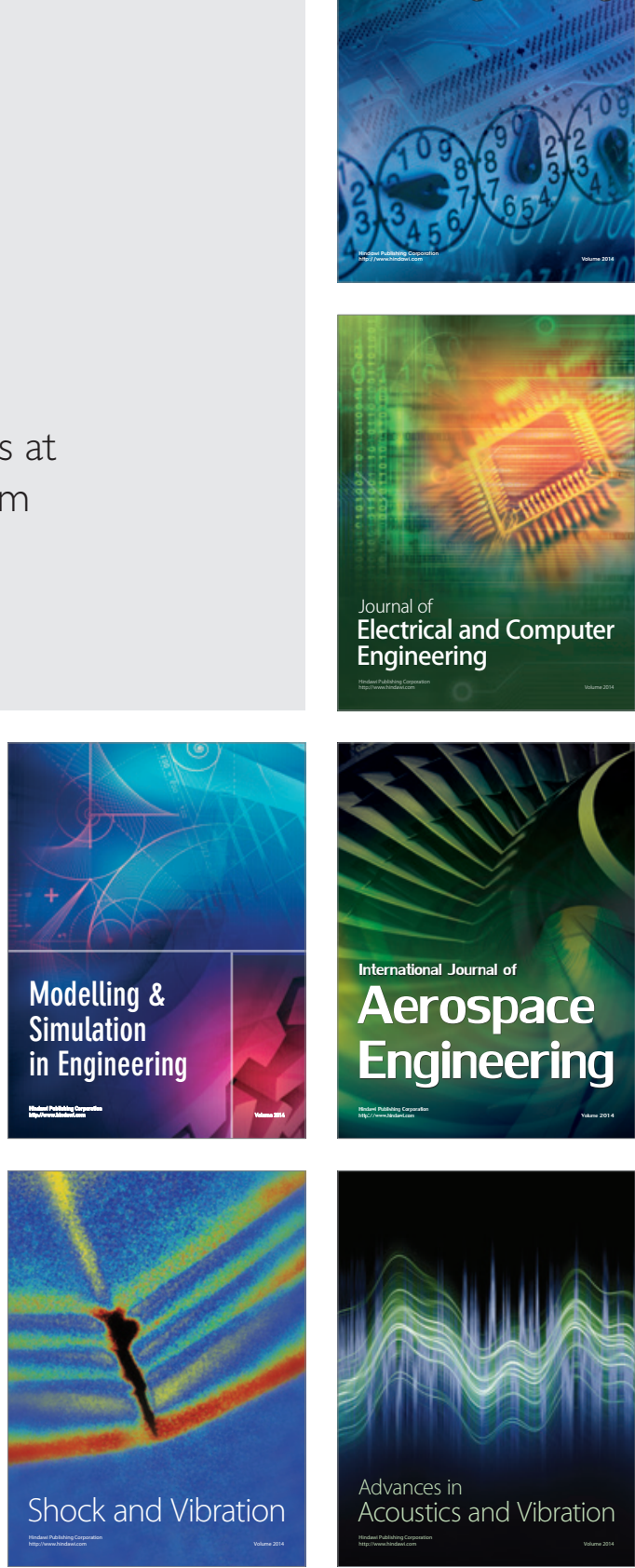